\title{
Inflammatory response in epilepsy is mediated by glial cell gap junction pathway (Review)
}

\author{
GUANGLIANG WANG ${ }^{1}$, JIANGTAO WANG ${ }^{2}$, CUIJUAN XIN ${ }^{2}$, \\ JINYU XIAO ${ }^{2}$, JIANMIN LIANG ${ }^{2}$ and XUEMEI WU ${ }^{2}$ \\ ${ }^{1}$ Department of Cardiology, Dalinghe Hospital of Far Eastern Horizon, Linghai, Liaoning 121200; \\ ${ }^{2}$ Department of Pediatric Neurology, Jilin University, Changchun, Jilin 130000, P.R. China
}

Received November 20, 2020; Accepted April 21, 2021

DOI: $10.3892 / \mathrm{mmr} .2021 .12132$

\begin{abstract}
Epilepsy is a common neurological disease that affects more than 50 million people worldwide. Neuroinflammation plays an important role in epilepsy. Activation of the immune system and an excessive inflammatory response can increase the frequency of seizures and increase the susceptibility to epilepsy. Therefore, anti-inflammatory therapies may have antiepileptic effects. Connexin 43 (Cx43) is a major component of astroglial hemichannels and gap junctions. Gap junctions are important for the direct exchange of substances and information between cells, as well as regulating the neuroinflammatory response, changing neuronal excitability, neuronal apoptosis, and synaptic remodeling. $\mathrm{Cx} 43$-mediated gap junction pathway can be crucial in epilepsy-induced neuroinflammatory cascades. Further, pro-inflammatory cytokines may in turn directly affect the expression of the $\mathrm{Cx} 43$ protein in astrocytes. Therefore, examining the association between neuroinflammation and epilepsy can be instrumental in uncovering the pathogenesis of epilepsy, which can lead to the development of novel and more effective antiepileptic drugs.
\end{abstract}

\section{Contents}

1. Introduction

2. Inflammatory reaction in the central nervous system

3. Role of the inflammatory reaction in the development of epilepsy

4. Regulation of astrocyte glial junction in epilepsy

5. Conclusion

Correspondence to: Dr Xuemei Wu, Department of Pediatric Neurology, Jilin University, 1 Xinmin Street, Changchun, Jilin 130000, P.R. China

E-mail: luoluo0914_cn@sina.com

Abbreviations: Cx43, connexin 43; ATP, adenosine triphosphate; $\mathrm{IL}$, interleukin; TNF, tumor necrosis factor

Key words: epilepsy, neuroinflammation, $\mathrm{Cx} 43$

\section{Introduction}

Epilepsy is a common neurological disease that affects $>50$ million people worldwide. Approximately $30 \%$ of epileptic patients are resistant to various antiepileptic drugs and eventually develop refractory epilepsy $(1,2)$. Although novel treatment strategies have been developed over the past few years, certain cases of refractory epilepsy cannot be controlled yet (3). In addition, most available treatments are focused on decreasing seizures by inhibiting the excitability of the nervous system (4). Therefore, the development of new antiepileptogenic therapies that can resolve seizures is imminent (5). Recent studies reported that neuroinflammation plays an important role in the development of epilepsy and abnormal neural circuits (6-11). Further, anti-inflammatory therapies were reported to have possible antiepileptogenic potential $(12,13)$. Therefore, uncovering the role of inflammatory reactions in epilepsy can lead to the development of more effective and novel antiepileptic drugs which can decrease the disability rate and improve the quality of life in epileptic patients.

\section{Inflammatory reaction in the central nervous system}

The central nervous system can be termed as an 'immunity exemption zone' because the blood-brain barrier restricts the entry of various substances in the blood circulation (14-17). However, accumulating evidence indicates that the immune exemption of the central nervous system is relative $(18,19)$. Engelhardt et al (20) reported that although immature T cells cannot pass through the blood-brain barrier, activated $\mathrm{T}$ cells can directly attach to the surface of the cerebral vascular endothelium and pass through the blood-brain barrier in the direction of blood flow. After crossing the blood-brain barrier, $\mathrm{T}$ cells can mediate inflammation in the central nervous system (20).

The central nervous system has a special 'immune defense line'. Cells involved in the brain's inflammatory reaction include the microglia, astrocytes and endothelial cells. Microglia are the immune sentinels of the central nervous system; they can be activated by injury stimuli and induce the corresponding inflammatory reaction, which maintains homeostasis in the central nervous system (21). The number of microglia is relatively small, accounting for $\sim 5 \%$ of the 


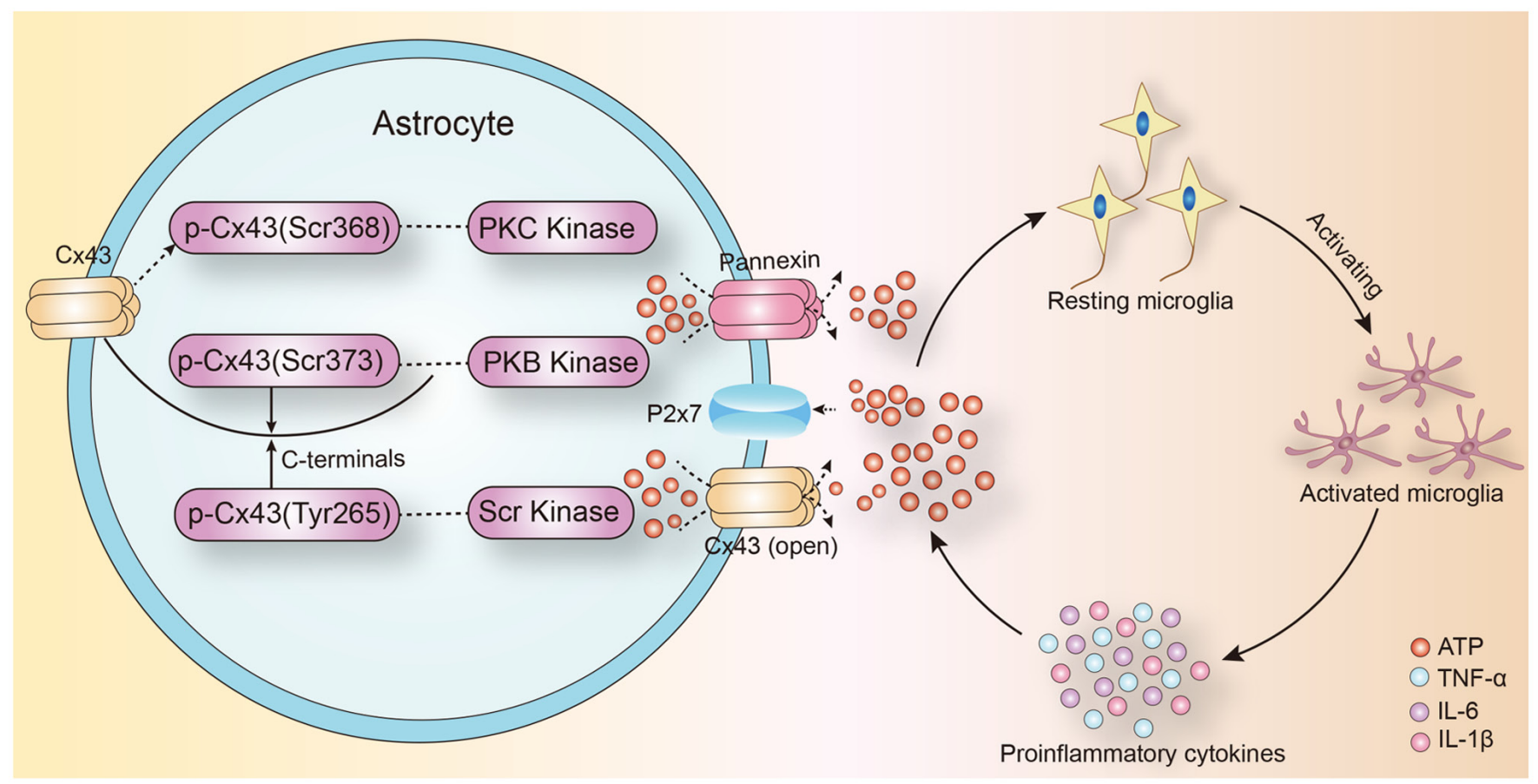

Figure 1. Proposed role of $\mathrm{Cx} 43$ pathway and ATP in inflammatory activation of microglia in epilepsy. Under physiological conditions, Cx43 is phosphorylated and the channel is closed. Cx43 is dephosphorylated by a variety of protein kinases or protein phosphorylases in epilepsy. The change in Cx43 conformation leads to abnormal opening of the channels. The release of the intracellular ATP mediates the activation and differentiation of microglia into proinflammatory phenotypes, and mediates the process of neuroinflammatory response in epilepsy. Cx43, connexin 43; TNF, tumor necrosis factor; IL, interleukin; ATP, adenosine triphosphate.

glial population (22-24). Following infection or damage to the central nervous system, the resting microglia acquire antigenicity, their shape becomes extended, and they are activated into macrophages to participate in the inflammatory reaction together with $\mathrm{T}$ cells inoculated within the blood circulation (25). However, hyperactivated microglia are important sources of pro-inflammatory factors as well as oxidative stress, and can cause neurotoxicity.

Astrocytes are also important contributors to the inflammatory reaction in the nervous system $(26,27)$. Astrocytes can play a role in neuronal migration movement, maintain the potassium concentration in the central nervous system, regulate neuronal excitability, and present antigens to autoreactive T cells.

Microglia are activated leading to the production of inflammatory factors following trauma or other injuries. Activated microglia can release cytotoxic substances and cytokines (28). Further, tissue injury could lead to the infiltration of circulating immune cells (29). Additionally, brain tissues are also exposed to systemic inflammatory response, which further aggravates the immune response and leads to secondary neuronal damage (30).

\section{Role of the inflammatory reaction in the development of epilepsy}

Activation of the immune system and an excessive inflammatory response play crucial roles in the development of chronic seizures (31-33). Moreover, neuronal inflammation associated with inflammatory cytokines signaling pathways may trigger epileptogenesis (34). Of note, patients diagnosed with relapsing remitting multiple sclerosis with epilepsy showed more extensive cortical inflammation compared with patients diagnosed with relapsing remitting multiple sclerosis without epilepsy (35). Therefore, examining the association between neuroinflammation and epilepsy can help uncover the pathogenesis. Clinical and animal studies suggested that the immune response is triggered during the pathophysiology of epilepsy and that the inflammatory reaction within the brain may be involved in the development of epilepsy $(36,37)$. Further, the dysregulation of immunoinflammatory reactions during the pathological course of epilepsy was associated with seizure-induced plasticity (10). Rana and Musto (37) reported that neuronal inflammation generated by neural death and astrocytes proliferation was associated with the microglial activation in damaged areas such as the amygdala, piriform and hippocampus in a rat model of lithium-pilocarpine-induced epilepsy (38). This dysfunction can facilitate the occurrence of epileptic seizures or epilepsy-induced neuronal damage (39). Indeed, inflammatory reactions were found to increase the propensity for seizures, change neuronal excitability, damage the blood-brain barrier, and mediate neuronal apoptosis and synaptic remodeling by activating intracellular signaling pathways (40). Seizures can activate microglia and neurons in the brain, and produce a series of inflammatory reactions without additional exogenous stimuli $(32,33)$. Consequently, microglia and neurons secrete large amounts of pro-inflammatory factors and prostaglandins, and activate the complement system, which ultimately promotes neuronal death and synapse regeneration, leading to chronic spontaneous seizures $(10,39)$.

Noteworthy, adenosine triphosphate (ATP) was shown to activate the sterile inflammatory process through interactions 
with purinergic receptors (40). The ATP-gated ionotropic $\mathrm{P} 2 \mathrm{X} 7$ receptor $(\mathrm{P} 2 \mathrm{X} 7 \mathrm{R})$ can mediate the regulation of neuroinflammation and immune reactions in the central nervous system (41). Neuronal injury was reported to activate microglia and increase the expression of P2X7R $(42,43)$. Monif et al (44) reported that the overexpression of P2X7R is sufficient to drive microglial activation. Moreover, the Fas ligand derived from microglia exacerbates P2X7-mediated microglial activation and triggers a vicious cycle of neuronal death $(45,46)$. Low concentrations of ATP act as chemotactic agents for microglia recognition and migration to guide them to the site of injury (47). ATP-stimulated and P2X4R-mediated microglial activation might have an initial protective effect. Activated microglia can remove potentially necrotic cell debris and promote tissue repair, thereby contributing to neuroprotection. Further, activated microglia release neurotrophic factors through activated P2X4Rs and contribute to neuronal survival (48). P2X7R-activated microglia in neuronmicroglia co-culture protect neurons from glutamate toxicity primarily by releasing tumor necrosis factor (TNF)- $\alpha$. The depletion of microglia can lead to an increase in the levels of cytokines and chemokines such as interleukin (IL)-1 $\beta$, TNF- $\alpha$, cytokine-induced neutrophil chemoattractant 1 and monocyte chemoattractant protein-1 in the brain, which aggravates brain damage (49). In later stages of brain injury, ATP can stimulate the overexpression of microglia $\mathrm{P} 2 \mathrm{X} 7 \mathrm{R}$, leading to microglia activation and proliferation, as well as cell death (50). Overactivated microglia upregulate the expression of surface immunomodulatory proteins and release of neurotoxic proinflammatory factors such as IL- $1 \beta$, IL- 6 , and TNF- $\alpha$, which can promote further activation of microglia. Long-term inflammatory reactions result in neuronal death that affects both healthy and damaged cells $(51,52)$.

The expression of connexin $43(\mathrm{Cx} 43)$ in astrocytes is affected by inflammatory cytokines (53). Treatment with IL-1 $\beta$, for $24 \mathrm{~h}$, inhibited the gap junction communication between the human embryonic astrocytes and decreased $\mathrm{Cx} 43$ mRNA and protein expression (54). IL-1 $\beta$ had a transient inhibitory effect on gap junction communication between primary astrocytes, and this inhibitory effect was produced through p38-dependent signaling pathway (55). Therefore, Cx43-mediated gap junction communication in astrocytes is closely correlated to the inflammatory response in the central nervous system. They regulate inflammation in the brain and selectively regulate the opening of gap junction communication by intracranial inflammatory factors (Fig. 1).

\section{Regulation of astrocyte glial junction in epilepsy}

Cx43 is a major component of astroglial hemichannels and gap junctions (56). Gap junctions play an important role in neuroinflammatory reactions (52). However, the impact of astrocyte Cx43, its hemichannel, and gap junctions on regulating the neuroinflammatory response in epilepsy is still unclear.

Astrocytes are the largest glial cell population in the central nervous system. $\mathrm{Cx} 43$ is a gap junction protein that is mainly expressed in astrocytes and mediates over 95\% of the gap junction communication in the brain (57). Under physiological conditions, gap junctions allow the exchange of small molecules $(<1.5 \mathrm{kDa})$ between cells. ATP mediates the migration of activated microglia to the injured area, especially in the initial phase of inflammation (41). Further, extracellular ATP induces the release of endogenous ATP from microglia and attracts distant microglia to the injury site, which leads to the promotion of the inflammatory cascade. ATP released by astrocyte hemichannels establishes an ATP gradient in the extracellular environment that can trigger microglial activation $(58,59)$. Increased extracellular ATP concentration in the injury site mediates the activation of microglia around the lesion (60). Of note, the local injection of ATP mimicked the traumatic brain injury-induced microglial activation and the administration of the gap junction channel blocker, carbenic acid, significantly inhibited the microglial activation $(61,62)$. Following injury, extracellular ATP is released from the open hemichannels and mediates a rapid reaction to microglial damage (63). Jesus et al (64) demonstrated that targeted knockout of astrocyte $\mathrm{Cx} 43$ expression decreased proinflammatory cytokine levels in the brain following lipopolysaccharide injection. In addition, hemichannel modulators like Cx43 mimetic peptide and $\mathrm{Cx}_{4} 43$ antisense oligonucleotides could inhibit the inflammatory response mediated by microglial activation following spinal cord injury (65). Taken together, it is plausible that astrocyte gap junction channel can act as a 'switch' in the inflammatory signaling cascade by promoting the release of ATP into the extracellular space. The inflammatory response can affect neuronal excitability, neuronal apoptosis and synaptic remodeling. These factors can lead to the development of abnormal neural excitability, which contributes to the pathogenesis of epilepsy. At the same time, pro-inflammatory cytokines can directly affect the expression of $\mathrm{Cx} 43$ protein in astrocytes (Fig. 1).

\section{Conclusion}

The occurrence, development and maintenance of epileptic seizures progress through a complicated process. The neuroinflammatory reaction can aggravate epilepsy and maintain recurrent episodes by increasing neuronal excitability, mediating neuronal apoptosis and remodeling the synapses. Therefore, controlling the neuroinflammatory reaction can mitigate the downstream cascade. The gap junction pathway mediated by astrocyte $\mathrm{Cx} 43$ can play a crucial role in controlling the epilepsy-induced neuroinflammatory cascade. Therefore, Cx43 can be a potential target for managing epileptic inflammatory reactions. Studies that examine the correlation between neuroinflammation and gap junctions will lead to a better understanding of epilepsy pathogenesis and can uncover new treatment targets.

\section{Acknowledgements}

Not applicable.

\section{Funding}

This study was funded by the National Natural Science Foundation of China (grant nos. 81801284, 81771396 and 31371125) and Jilin Provincial Ring-fenced Funding for Industrial Innovation Project (grant no. 2017C029-1). 


\section{Availability of data and materials}

Not applicable.

\section{Authors' contributions}

CX and JX searched for literature; GW and JW wrote this review; JL and XW revised this review. All authors read and approved the final manuscript. Data authentication is not applicable.

\section{Ethics approval and consent to participate}

Not applicable.

\section{Patient consent for publication}

Not applicable.

\section{Competing interests}

The authors declare that they have no competing interests.

\section{References}

1. Beghi E and Giussani G: Treatment of epilepsy in light of the most recent advances. Lancet Neurol 18: 7-8, 2019.

2. Brewer MK, Grossman TR, McKnight TR, Goldberg YP, Landy $\mathrm{H}$ and Gentry MS: The 4th International Lafora Epilepsy Workshop: Shifting paradigms, paths to treatment, and hope for patients. Epilepsy Behav 90: 284-286, 2019.

3. Conway CR, Udaiyar A and Schachter SC: Neurostimulation for depression in epilepsy. Epilepsy Behav 88S: 25-32, 2018.

4. Johnson EL: Seizures and epilepsy. Med Clin North Am 103 309-324, 2019.

5. Klein P,Dingledine R, Aronica E, Bernard C, Blümcke I, Boison D, Brodie MJ, Brooks-Kayal AR, Engel J Jr, Forcelli PA, et al: Commonalities in epileptogenic processes from different acute brain insults: Do they translate? Epilepsia 59: 37-66, 2018.

6. Vezzani A, Balosso S and Ravizza T: Neuroinflammatory pathways as treatment targets and biomarkers in epilepsy. Nat Rev Neurol 15: 459-472, 2019.

7. Hodges SL and Lugo JN: Therapeutic role of targeting mTOR signaling and neuroinflammation in epilepsy. Epilepsy Res 161: 106282, 2020.

8. Eastman CL, D'Ambrosio R and Ganesh T: Modulating neuroinflammation and oxidative stress to prevent epilepsy and improve outcomes after traumatic brain injury. Neuropharmacology 172 : 107907, 2020

9. Terrone G, Balosso S, Pauletti A, Ravizza T and Vezzani A: Inflammation and reactive oxygen species as disease modifiers in epilepsy. Neuropharmacology 167: 107742, 2020.

10. Sanz P and Garcia-Gimeno MA: Reactive glia inflammatory signaling pathways and epilepsy. Int J Mol Sci 21: 4096, 2020.

11. Mukherjee S, Arisi GM, Mims K, Hollingsworth G, O'Neil K and Shapiro LA: Neuroinflammatory mechanisms of post-traumatic epilepsy. J Neuroinflammation 17: 193, 2020.

12. Korff CM and Dale RC: The immune system in pediatric seizures and epilepsies. Pediatrics 140: e20163534, 2017.

13. Bauer J, Becker AJ, Elyaman W, Peltola J, Rüegg S, Titulaer MJ, Varley JA and Beghi E: Innate and adaptive immunity in human epilepsies. Epilepsia 58 (Suppl 3): 57-68, 2017

14. Tamura R, Yoshida K and Toda M: Current understanding of lymphatic vessels in the central nervous system. Neurosurg Rev 43: 1055-1064, 2020.

15. Kipnis J: Multifaceted interactions between adaptive immunity and the central nervous system. Science 353: 766-771, 2016.

16. Klein RS, Garber $\mathrm{C}$ and Howard N: Infectious immunity in the central nervous system and brain function. Nat Immunol 18: 132-141, 2017.
17. Wesselingh R, Butzkueven H, Buzzard K, Tarlinton D, O'Brien TJ and Monif M: Innate immunity in the central nervous system: A missing piece of the autoimmune encephalitis puzzle? Front Immunol 10: 2066, 2019.

18. Peralta Ramos JM, Iribarren P, Bousset L, Melki R, Baekelandt V and Van der Perren A: Peripheral inflammation regulates CNS immune surveillance through the recruitment of inflammatory monocytes upon systemic $\alpha$-synuclein administration. Front Immunol 10: 80, 2019.

19. Prinz M and Priller J: The role of peripheral immune cells in the CNS in steady state and disease. Nat Neurosci 20: 136-144, 2017.

20. Engelhardt B, Vajkoczy P and Weller RO: The movers and shapers in immune privilege of the CNS. Nat Immunol 18: 123-131, 2017.

21. Greenhalgh AD, Zarruk JG, Healy LM, Baskar Jesudasan SJ, Jhelum P, Salmon CK, Formanek A, Russo MV, Antel JP, McGavern DB, et al: Peripherally derived macrophages modulate microglial function to reduce inflammation after CNS injury. PLoS Biol 16: e2005264, 2018.

22. Bohlen CJ, Friedman BA, Dejanovic B and Sheng M: Microglia in brain development, homeostasis, and neurodegeneration. Annu Rev Genet 53: 263-288, 2019.

23. Tan YL, Yuan Y and Tian L: Microglial regional heterogeneity and its role in the brain. Mol Psychiatry 25: 351-367, 2020.

24. Illes P, Rubini P, Ulrich H, Zhao Y and Tang Y: Regulation of microglial functions by purinergic mechanisms in the healthy and diseased CNS. Cells 9: 1108, 2020.

25. Hsu M, Rayasam A, Kijak JA, Choi YH, Harding JS, Marcus SA, Karpus WJ, Sandor M and Fabry Z: Neuroinflammation-induced lymphangiogenesis near the cribriform plate contributes to drainage of CNS-derived antigens and immune cells. Nat Commun 10: 229, 2019.

26. Wheeler MA, Jaronen M, Covacu R, Zandee SEJ, Scalisi G, Rothhammer V, Tjon EC, Chao CC, Kenison JE, Blain M, et al: Environmental control of astrocyte pathogenic activities in CNS inflammation. Cell 176: 581-596.e18, 2019.

27. Rothhammer V and Quintana FJ: Control of autoimmune CNS inflammation by astrocytes. Semin Immunopathol 37: 625-638, 2015.

28. Anderson WD, Greenhalgh AD, Takwale A, David S and Vadigepalli R: Novel influences of IL-10 on CNS inflammation revealed by integrated analyses of cytokine networks and microglial morphology. Front Cell Neurosci 11: 233, 2017.

29. Chiang N and Serhan CN: Structural elucidation and physiologic functions of specialized pro-resolving mediators and their receptors. Mol Aspects Med 58: 114-129, 2017.

30. Dupuis N and Auvin S: Inflammation and epilepsy in the developing brain: Clinical and experimental evidence. CNS Neurosci Ther 21: 141-151, 2015.

31. Mukhtar I: Inflammatory and immune mechanisms underlying epileptogenesis and epilepsy: From pathogenesis to treatment target. Seizure 82: 65-79, 2020.

32. Wyatt-Johnson SK and Brewster AL: Emerging Roles for Microglial Phagocytic Signaling in Epilepsy. Epilepsy Curr 20: 33-38, 2020.

33. Hiragi T, Ikegaya $Y$ and Koyama R: Microglia after seizures and in epilepsy. Cells 7: 26, 2018

34. Paudel YN, Shaikh MF, Shah S, Kumari Y and Othman I: Role of inflammation in epilepsy and neurobehavioral comorbidities: Implication for therapy. Eur J Pharmacol 837: 145-155, 2018.

35. Calabrese M, De Stefano N, Atzori M, Bernardi V, Mattisi I, Barachino L, Rinaldi L, Morra A, McAuliffe MM, Perini P, et al: Extensive cortical inflammation is associated with epilepsy in multiple sclerosis. J Neurol 255: 581-586, 2008.

36. Drion CM, van Scheppingen J, Arena A, Geijtenbeek KW, Kooijman L, van Vliet EA, Aronica E and Gorter JA: Effects of rapamycin and curcumin on inflammation and oxidative stress in vitro and in vivo - in search of potential anti-epileptogenic strategies for temporal lobe epilepsy. J Neuroinflammation 15: 212,2018

37. Rana A and Musto AE: The role of inflammation in the development of epilepsy. J Neuroinflammation 15: 144, 2018.

38. Jung KH, Chu K, Lee ST, Kim JH, Kang KM, Song EC, Kim SJ, Park HK, Kim M, Lee SK, et al: Region-specific plasticity in the epileptic rat brain: A hippocampal and extrahippocampal analysis. Epilepsia 50: 537-549, 2009.

39. Vezzani A and Rüegg S: Introduction to the 2nd Meeting on Immunity and Inflammation in Epilepsy (IIE2016). Epilepsia 58 (Suppl 3): 7-10, 2017.

40. Mazarati AM, Lewis ML and Pittman QJ: Neurobehavioral comorbidities of epilepsy: Role of inflammation. Epilepsia 58 (Suppl 3): 48-56, 2017. 
41. Allsopp RC, Dayl S, Schmid R and Evans RJ: Unique residues in the ATP gated human P2X7 receptor define a novel allosteric binding pocket for the selective antagonist AZ10606120. Sci Rep 7: 725, 2017.

42. Nuka E, Ohnishi K, Terao J and Kawai Y: ATP/P2X7 receptor signaling as a potential anti-inflammatory target of natural polyphenols. PLoS One 13: e0204229, 2018.

43. Zumerle S, Calì B, Munari F, Angioni R, Di Virgilio F, Molon B and Viola A: Intercellular calcium signaling induced by ATP potentiates macrophage phagocytosis. Cell Rep 27: 1-10.e4, 2019.

44. Monif M, Burnstock G and Williams DA: Microglia: Proliferation and activation driven by the P2X7 receptor. Int J Biochem Cell Biol 42: 1753-1756, 2010

45. Li R, Shang Y, Hu X, Yu Y, Zhou T, Xiong W and Zou X: ATP $\mathrm{P} 2 \mathrm{X} 7 \mathrm{r}$ axis mediates the pathological process of allergic asthma by inducing M2 polarization of alveolar macrophages. Exp Cell Res 386: 111708, 2020.

46. Ryoden Y, Fujii T, Segawa K and Nagata S: Functional expression of the P2X7 ATP receptor requires eros. J Immunol 204: 559-568, 2020.

47. Burnstock G and Knight GE: The potential of P2X7 receptors as a therapeutic target, including inflammation and tumour progression. Purinergic Signal 14: 1-18, 2018.

48. Amadio S, Parisi C, Piras E, Fabbrizio P, Apolloni S, Montilli C, Luchetti S, Ruggieri S, Gasperini C, Laghi-Pasini F, et al: Modulation of $\mathrm{P} 2 \mathrm{X} 7$ receptor during inflammation in multiple sclerosis. Front Immunol 8: 1529, 2017.

49. Wei L, Syed Mortadza SA, Yan J, Zhang L, Wang L, Yin Y, Li C, Chalon S, Emond P, Belzung C, et al: ATP-activated P2X7 receptor in the pathophysiology of mood disorders and as an emerging target for the development of novel antidepressant therapeutics. Neurosci Biobehav Rev 87: 192-205, 2018.

50. Beamer E, Fischer W and Engel T: The ATP-gated P2X7 receptor as a target for the treatment of drug-resistant epilepsy. Front Neurosci 11: 21, 2017.

51. Kaczmarek-Hajek K, Zhang J, Kopp R, Grosche A, Rissiek B, Saul A, Bruzzone S, Engel T, Jooss T, Krautloher A, et al: Re-evaluation of neuronal P2X7 expression using novel mouse models and a P2X7-specific nanobody. Elife 7: e36217, 2018.

52. Kim Y, Davidson JO, Gunn KC, Phillips AR, Green CR and Gunn AJ: Role of hemichannels in CNS inflammation and the inflammasome pathway. Adv Protein Chem Struct Biol 104: 1-37, 2016.

53. Das A, Wallace GC IV, Holmes C, McDowell ML, Smith JA, Marshall JD, Bonilha L, Edwards JC, Glazier SS, Ray SK, et al: Hippocampal tissue of patients with refractory temporal lobe epilepsy is associated with astrocyte activation, inflammation, and altered expression of channels and receptors. Neuroscience 220: 237-246, 2012
54. John GR, Scemes E, Suadicani SO, Liu JS, Charles PC, Lee SC, Spray DC and Brosnan CF: IL-1beta differentially regulates calcium wave propagation between primary human fetal astrocytes via pathways involving $\mathrm{P} 2$ receptors and gap junction channels. Proc Natl Acad Sci USA 96: 11613-11618, 1999.

55. Zvalova D, Cordier J, Mesnil M, Junier MP and Chneiweiss H: p38/SAPK2 controls gap junction closure in astrocytes. Glia 46: 323-333, 2004

56. De Bock M, Wang N, Decrock E, Bultynck G and Leybaert L: Intracellular cleavage of the Cx43 C-terminal domain by matrix-metalloproteases: A novel contributor to inflammation? Mediators Inflamm 2015: 257471, 2015.

57. Jourdeuil $\mathrm{K}$ and Taneyhill LA: The gap junction protein connexin 43 controls multiple aspects of cranial neural crest cell development. J Cell Sci 133: jcs235440, 2020.

58. Nikolic L, Shen W, Nobili P, Virenque A, Ulmann L and Audinat E: Blocking TNF $\alpha$-driven astrocyte purinergic signaling restores normal synaptic activity during epileptogenesis. Glia 66 : 2673-2683, 2018.

59. Ghaemi A, Alizadeh L, Babaei S, Jafarian M, Khaleghi Ghadiri M, Meuth SG, Kovac S and Gorji A: Astrocyte-mediated inflammation in cortical spreading depression. Cephalalgia 38: 626-638, 2018

60. Webster KM, Sun M, Crack P, O'Brien TJ, Shultz SR and Semple BD: Inflammation in epileptogenesis after traumatic brain injury. J Neuroinflammation 14: 10, 2017.

61. Illes P, Burnstock G and Tang Y: Astroglia-derived ATP modulates CNS neuronal circuits. Trends Neurosci 42: 885-898, 2019.

62. Nakamura Y, Park JH and Hayakawa K: Therapeutic use of extracellular mitochondria in CNS injury and disease. Exp Neurol 324: 113114, 2020.

63. Li W, Li J, Sama AE and Wang H: Carbenoxolone blocks endotoxin-induced protein kinase R (PKR) activation and high mobility group box 1 (HMGB1) release. Mol Med 19: 203-211, 2013.

64. Jesus LB, Santos AB, Jesus EEV, Santos RGD, Grangeiro MS, Bispo-da-Silva A, Arruda MR, Argolo DS, Pinheiro AM, El-Bachá RS, et al: IDO, COX and iNOS have an important role in the proliferation of Neospora caninum in neuron/glia co-cultures. Vet Parasitol 266: 96-102, 2019.

65. Kwan T, Floyd CL, Kim S and King PH: RNA binding protein human antigen $\mathrm{R}$ is translocated in astrocytes following spinal cord injury and promotes the inflammatory response. J Neurotrauma 34: 1249-1259, 2017.

(i) $($ ) This work is licensed under a Creative Commons Attribution-NonCommercial-NoDerivatives 4.0 International (CC BY-NC-ND 4.0) License. 